\title{
On a Generalized Model in Accelerated Life Testing
}

\author{
Eduardo L. Cruz ${ }^{1}$, Adolfo M. De Guzman ${ }^{2}$ \\ ${ }^{1}$ Department of Epidemiology and Biostatistics, University of the Philippines, Manila, Philippines \\ ${ }^{2}$ School of Statistics, University of the Philippines, Quezon City, Philippines \\ Email: edcruz2@up.edu.ph
}

Received January 30, 2012; revised February 29, 2012; accepted March 14, 2012

\begin{abstract}
The main objective of accelerated life tests in this setting is the recovery of the distribution of the random variable representing lifetime which is difficult to observe at a certain level of a given stress factor. A general model for accelerated life test is proposed that utilizes the inverse problem approach, that is, the variable is observe at different level/s and the transfer function is used to recover the elusive random variable (life time). The problem then is reduced to finding the transfer function. We derive some properties of the proposed general model. The Lognormal distribution and the Arrhenius model for lifetime are used as examples. Its relationship with the Cox proportional hazards model is also discussed.
\end{abstract}

Keywords: Accelerated Life Test; Arrhenius Model; Inverse Problem

\section{Introduction}

From the point of view of production and reliability engineers, accelerated life testing is an important aspect of product development, quality control and improvement. Accelerated life testing is accomplished by applying increased stress on the product or product component. It is intended to produce data on strength and on lifetime of material components and systems. For an excellent but elementary exposition on this, the reader is referred to Nelson [1].

Accelerated life testing is also very interesting from the point of view of theory. Most lifetime data suffer from the censoring problem of statistics. Lifetime observations usually exceed the interest time of the observer or even his own lifetime. Accelerated life tests, however, are performed to destruction thereby eliminating censored observations. The analysis of censored or incomplete data requires a modification of the usual statistical methodology of independent and identically distributed observations and their uncensored generalizations. Recent developments point to the counting process approach with the use of martingales as introduced by Aalen [2] for analyzing survival or lifetime data. The audience is referred to Anderson, Borgen, Gill and Keiding [3] and Fleming and Harrington [4] for a comprehensive treatment. The first volume covers extensively both theory and applications of the counting process approach to survival analysis while the second volume treats the analysis of clinical data via the martingale approach. Both books require some amount of mathematical as well as statisti- cal sophistication. It takes more time for this approach to be a practical statistical technology. An alternative to the counting approach is provided by the Accelerated Life Testing in some interesting cases. This is so because it reduces the number of incomplete observations and even eliminate them.

Section 2 of this paper describes a generalized model for accelerated life testing. Its properties are stated and discussed in Section 3 where some examples are also provided. Section 4 gives some concluding remarks and future work.

\section{A Generalized Model for Accelerated Life Test}

Let $X$ be a nonnegative random variable that represents lifetime in the normal condition, that is, without any stress applied to it, with unknown distribution $F$. Suppose stress is applied on $X$ and the observations say,

$X_{1 s}, X_{2 s}, \cdots, X_{n s}$ are made from a distribution, say $F_{s}$. This setting is an example of the inverse problem approach in statistics where the stress $S$ is a known operator. That is,

$$
S: X \rightarrow X_{S} \text { or } S: F \rightarrow F_{S},
$$

in some space of random variables. Here the stress $s$ may have $K$ levels, i.e. $s_{1}, s_{2}, \cdots, s_{K}$.

An approach to accelerated life test model is given by Cox and Oakes [5] and Barlow and Sheuer [6] and also appears in Gnedenko, et al. [7]. This is described as follows. Denote a failure time distribution function under a 
normal stress condition by $F_{0}(\cdot)$. The accelerated life time transformation is given in terms of $F(t, z)$ and $F_{0}(\cdot)$ by the relationship

$$
F(t, z)=F_{0}[t \psi(z, A)],
$$

where $\psi(z, A)$ is a positive function (or acceleration function) connecting "time to failure" with a stress factor $z$, and $A$ is a vector of unknown parameters. For $z=0$, $\psi(z, A)$ is assumed equal to 1 . This relationship is a scale transformation where a change in stress does not result in a change in the shape of the distribution function but changes its scale only. This relationship can be written in terms of the acceleration function as

$$
g(t)=\psi(z, A) t .
$$

In other words, the relationship above is linear with a time acceleration function $\psi$.

We now propose a model for accelerated life test generalizes the relationship in (3). This general model is expressed in terms of random variables and thus is simpler in structure. Let $S$ be a nonempty set whose elements we will refer to as stress space. For example, in the Arrhenius model, $S$ is the set of nonnegative real numbers representing temperature. We define the proposed model by

$$
X_{s}=A(s, \theta) X,
$$

where $X$ is the random variable under normal use, $\theta$ is a vector of parameters, $s \in S$ and $A(s, \theta)$ is called the scaling or acceleration function which is a monotone function continuous from the right. This model is a stochastic process $\left\{X_{S}: s \in S\right\}$ and will be called a general lifetime model with stress space $S$. The model says the random lifetime $X_{S}$ depends on the stress $s$, where stress is a state or configuration of stress factors belonging to some known stress space $S$. Information on the stress space should be available in the underlying field such as medicine for clinical trials, psychology for behavioral studies, chemistry for phenomena depending on clinical reactions, to name a few.

Two situations arise from this model: first, if stress $s$ is fixed or controlled, this model is interpreted as a general accelerated life test model. If $s$ is random, the model is known as the survival model with covariates. In this paper we consider only the first case where stress $s$ is fixed. We will refer to this model as the Generalized Model for Accelerated Life Testing (GMALT). It will be shown how this model relates to the well-known Arrhenius model in reliability engineering. The Arrhenius model is widely used to model product life as a function of temperature. Applications include electrical insulations, solid state and semiconductor devices, battery cells, and incandescent lamp filaments.

\section{Elementary Properties of the GMALT and Some Examples}

As mentioned in the introduction, the main advantage of the proposed model which is expressed as a scaling of random variables is that its properties can be studied more conveniently. In this section we present some analytic properties of the proposed model and state them as propositions.

\subsection{Mean and Covariance Function}

Proposition 1. If $X_{S}$ is a gmalt with acceleration function $A(s, \theta)$ and stress space $S$, then the mean function $\mu_{\mathrm{s}}$ and the covariance function $\sigma_{(s, t)}^{2}$ are given by,

$$
\begin{aligned}
\mu(s) & =A(s, \theta) E(X)=A(s, \theta) \mu_{X}, \quad s \in S \text { and } \\
\sigma_{(s, t)}^{2} & =E\left[\left(A(s, \theta) X-A(s, \theta) \mu_{X}\right)-A(t, \theta) \mu_{X}\right] \\
& =A(s, \theta) A(t, \theta) \sigma_{X}^{2},
\end{aligned}
$$

where $\mu_{X}$ and $\sigma_{X}^{2}>0$ are the mean and variance of the untransformed $X$, respectively. In particular, the variance at any stress $s \in S$ is $\sigma_{(s)}^{2}=[A(s, \theta)]^{2} \sigma_{X}^{2}$. The proof of these is straightforward and follows from the properties of expectation. To keep things simple, we write $A(s)$ instead of $A(s, \theta)$ from now on.

The next property shows the effect of log transformation in the GMALT model. The proof follows directly from applying the definition of logarithmic function and mathematical expectation.

\subsection{Effect of Log Transformation}

Proposition 2. If $X_{S}=A(s) X$, then $\log \left(X_{S}\right)$ has constant variance $\sigma^{2}=\operatorname{Var}\left(\log X_{S}\right)$ and mean $\tilde{\mu}$ given by

$$
\tilde{\mu}=\mu_{0}+\mu_{\log },
$$

where $\mu_{0}=\log [A(s)]$ and $\mu_{\log }=E(\log X)$.

So the log transformation results in a constant variance but a changing mean. In particular, since $A(s) \in(0,1]$, the mean decreases since $\log A(s) \leq 0$. An interesting case is when $\log \left(X_{S}\right)$ has a Normal distribution.

Example 1. (Lognormal model) In the GMALT, $X_{S}$ is Lognormal with mean $\tilde{\mu}_{S}$ and variance $\tilde{\sigma}_{S}^{2}$ if $\log X_{s}$ is normal with mean, say $\tilde{\mu}$ and variance $\tilde{\sigma}^{2}$. In this case the parameters of $X_{S}$ are $\tilde{\mu}_{S}=b \exp \left(\tilde{\sigma}^{2} / 2\right)$ and $\tilde{\sigma}_{S}^{2}=b^{2} \exp \left(\tilde{\sigma}^{2}\right)\left[\exp \left(\tilde{\sigma}^{2}-1\right)\right]$ where $b=\exp (\tilde{\mu})$, and $\tilde{\mu}$ and $\tilde{\sigma}^{2}$ are as defined in Property 2 (See Hogg and Craig [8] for properties of Lognormal distribution).

This example will enable us to make inferences about $X$ which is not observed in the GMALT model via the acceleration function $A(s)$, when it is known.

In the next example we show a situation where the 
model in Equation (4) fails. This example is also used to verify the second property which states the effect of log transformation on the GMALT.

Example 2. The data in Table 1 are hours to turn failure of a new Class-H insulation system tested in Motorettes at 190, 220, 240, and 260 Temperature $\left({ }^{\circ} \mathrm{C}\right)$. The original purpose of the experiment was to estimate the median time to turn failure at the design of 180 Temperature $\left({ }^{\circ} \mathrm{C}\right)[1]$.

To verify the second property in Section 3.2, we compare the variances of the untransformed and the logtransformed gmalt model. But we will see that Property 2 is satisfied on the three temperature levels only, as the stress level 260 Temperature $\left({ }^{\circ} \mathrm{C}\right)$ seemed to have violated an assumption of accelerated life model. In Tables 2 and 3 we see that the variances of both the untransformed and the log-transformed lifetime at all temperature levels are statistically different, but is not so for the first three levels, where the variances of the log-transformed data are not statistically different. Our observation, is that failure

Table 1. Hours to failure in an accelerated life test of class $\mathbf{H}$ insulation in Motorettes.

\begin{tabular}{cccc}
\hline \multicolumn{4}{c}{ Temperature $\left({ }^{\circ} \mathrm{C}\right)$ Levels } \\
\hline $190^{\circ} \mathrm{C}$ & $220^{\circ} \mathrm{C}$ & $240^{\circ} \mathrm{C}$ & $260^{\circ} \mathrm{C}$ \\
7228 & 1764 & 1175 & 600 \\
7228 & 2436 & 1175 & 744 \\
7228 & 2436 & 1521 & 744 \\
8448 & 2436 & 1569 & 744 \\
9167 & 2436 & 1617 & 912 \\
9167 & 2436 & 1665 & 1128 \\
9167 & 3108 & 1665 & 1320 \\
9167 & 3108 & 1713 & 1464 \\
10511 & 3108 & 1761 & 1608 \\
10511 & 3108 & 1953 & 1896 \\
\hline
\end{tabular}

Table 2. Test of homogenity of variance (all temperature levels) of hours to failure and its log transformation Motorettes data.

\begin{tabular}{ccc}
\hline Life time of Motorette & Levene Statistic & Significance \\
\hline Hours to failure & 9.962 & 0.000 \\
Log (Hours to failure) & 8.373 & 0.000 \\
\hline
\end{tabular}

Table 3. Test of homogenity of variance (190, 220 and 240 Temperature $\left({ }^{\circ} \mathrm{C}\right.$ ) levels only) of hours to failure and its $\log$ transformation in class $\mathbf{H}$ insulation in Motorettes data.

\begin{tabular}{ccc}
\hline Life time of Motorette & Levene Statistic & Significance \\
\hline Hours to failure & 7.708 & 0.001 \\
Log (Hours to failure) & 0.712 & 0.553 \\
\hline
\end{tabular}

modes are different at these two sets of levels (the 190, 220 and 240 Temperature $\left({ }^{\circ} \mathrm{C}\right)$ degrees, and the 260 Temperature $\left({ }^{\circ} \mathrm{C}\right)$ ), and that the stress level 260 Temperature $\left({ }^{\circ} \mathrm{C}\right)$ may have destroyed the shape of the distribution, thus violating the scaling assumption of the model, where a change in stress does not result in a change in the shape of the distribution function but changes its scale only. So, in this example we see that for the first three temperature levels, the log transformation has resulted in a constant variance but with still different (decreasing) mean, thus verifying Property 2. For this example, therefore the proposed model is applicable on the first three stress levels only. The following will be useful in interpreting the log mean of $X_{S}$ and $X$.

Proposition 3. If $Z$ is a $\log$ symmetric random variable with $\log$ mean $\tilde{\mu}_{S}$, i.e. $E(\log (Z))=\tilde{\mu}_{S}$, then $\exp \left(\tilde{\mu}_{S}\right)=\operatorname{median}(Z)$, where $\tilde{\mu}_{S}$ is as in Example 1 .

Proof of Proposition 3. From symmetry we have

$$
P\left(\log (Z) \leq \tilde{\mu}_{S}\right)=1 / 2 \text {. }
$$

Since

$$
P\left(\log (Z) \leq \tilde{\mu}_{S}\right)=P\left[Z \leq \exp \left(\tilde{\mu}_{S}\right)\right]
$$

then $F\left[\exp \left(\tilde{\mu}_{S}\right)\right]=F[\operatorname{median}(Z)]$, where $F$ is the distribution function of $Z$.

The result follows for absolutely continuous distribution functions $F$. We next relate the mean $\tilde{\mu}$ and variance $\tilde{\sigma}^{2}$ to the mean and variance of the untransformed or "normal use" random variable $X$. To do that we need the next result.

Proposition 4. Let $X$ be a any nonnegative random variable with distribution function $F$ and finite mean $\mu_{F}=E(X)$ and second moment $E\left(X^{2}\right)$. Then there exists positive numbers $\delta_{F}^{2}$ and $\gamma_{F}$ such that, $E(\log X) \leq \gamma_{F}$ and $\operatorname{Var}(\log X) \geq \delta_{F}^{2}+\left(\gamma_{F}-\mu_{F}^{2}\right)^{2}$. In fact, $\gamma_{F}=\int_{1}^{\infty} x \mathrm{~d} F(x)$ and $\gamma_{F}^{2}=\int_{0}^{y} x^{2} \mathrm{~d} F(x)$ where $y=-\log x$. We may now state the next property.

Proposition 5. In the GMALT model with acceleration function $A(s) \in(0,1)$ and stress space $S$, the expectations and variance of the log transformation of $X_{S}$ have the properties

$$
E\left[\log X_{S}\right] \leq \log A(s)+\gamma_{F} \leq \gamma_{F},
$$

and

$$
\operatorname{Var}\left(\log X_{S}\right) \geq \delta_{F}^{2}-\gamma_{F},
$$

where the random variable $X$ with distribution $F$ satisfies the usual regularity conditions and $\delta_{F}^{2}$ and $\gamma_{F}$, are as in Proposition 4.

The next result states the equivalence of our approach to the classical Arrhenius model where the acceleration function is given by 


$$
A(s)=\exp \left(\alpha_{1}-\alpha_{2} / s\right)
$$

with $s \in(0, \infty)$ is a function of temperature and $\alpha_{1}$ and $\alpha_{2}$ are constants characteristic of the product failure mechanism and test conditions [9].

\subsection{Relationship to Cox Proportional Hazards Model and Arrhenius Model}

In the next result we show the equivalence of the GMALT model and the Cox regression model or the proportional hazards model. The reader is referred to Fleming and Harrington [4] for an exposition. This result shows that the dual of the GMALT model is the Cox proportional hazards in the counting process approach to survival analysis, the GMALT being the lifetime approach to survival analysis. The Arhennius life model is also presented as special case.

Proposition 6. In the GMALT model with acceleration function $A(s)$ with stress space $S$, the hazard function at any stress $s \in S$ is given by

$$
\lambda(x, s)=A(s)^{-1} \lambda_{0}(x) \text { for all } x \in(0, \infty)
$$

If $A(s)=\exp \left(-\beta_{0}-\beta_{1} X\right)$ where $X$ is the covariate, then $\lambda(x, s)=\lambda_{0}(x) \exp \left(\beta_{0}+\beta_{1} X\right)$ which is the $\operatorname{Cox}$ proportional hazards model with one covariate. In particular, if $X=s^{-1}, \beta_{0}=-\alpha_{1}$ and $\beta_{1}=-\alpha_{2}$ where $s$ is temperature, and $\alpha_{1}$ and $\alpha_{2}$ are constants, we get the well-known Arhennius model [9].

Proof of Proposition 6. Let $P\left(X_{S} \leq y\right)=F_{S}$. Then

$$
\begin{aligned}
F_{S} & =P(A(s) X \leq y)=P[X \leq(A(s) / y)], \\
& =F_{X}[y / A(s)],
\end{aligned}
$$

where $F_{X}$ is the distribution of $X$. Also

$\partial F_{X}(y / A(s))=A(s)^{-1} f_{X}(y / A(s))$ where $f_{X}$ is the density of $X$. By definition the hazard function (at a given stress level $s$ ) is

$$
\begin{aligned}
\lambda(x, s) & =\frac{f_{S}(y)}{\left[1-F_{S}(y)\right]}=\frac{A(s)^{-1} f_{X}(y / A(s))}{\left[1-F_{X}(y / A(s))\right]} \\
& =A(s)^{-1} \lambda_{0}(x),
\end{aligned}
$$

by letting $x=y / A(s)$.

\subsection{Equivalence to Model of Cox and Oakes}

Finally we show the equivalence of the proposed model

(4) to the one given by Cox and Oakes [5] and described in Section 2.

Let $X$ have distribution function $F$. Since

$X_{S}=A(s, \theta) X$ we have

$$
\begin{aligned}
P\left(X_{S} \leq t\right) & =P(A(s, \theta) X \leq t)=P(X \leq(t / A(s, \theta)) \\
& =F[t / A(s, \theta)] .
\end{aligned}
$$

Taking $A(s, \theta)=1 / \psi(s, \theta)$ gives $F(t ; s)=F[\psi(s, \theta) t]$ and the two are equivalent. Thus, the approach of Cox and Oakes may be also taken as a special case of GMALT.

\section{Concluding Remarks}

In this paper, we considered a general model for accelerated life testing and derive some of its properties. This model is expressed in terms of the random variables which is simpler, instead of distribution functions. An important consideration is the choice of stress level, where the GMALT model may fail. A threshold level (stress) at which the scaling is no longer applicable must be sought by the scientist. Future work may consider where a test of hypothesis $H_{0}: A(s) \geq 1$ applies. Rejection of this hypothesis means we can proceed to the test or increase stress at a certain level. For the simple null hypothesis $H_{0}: A(s)=1$, rejection in favor of the alternative $H_{0}: A(s)<1$ indicates that we can start doing accelerated testing at stress level $s$ where $A(s)<1$. For products where $A(s)$ is not known, the search for $A(s)$ is the subject of many inquiries.

\section{Acknowledgements}

E. L. C. thanks the Office of the Vice President for Academic Affairs of the University of the Philippines where he was recipient of Doctoral Student Grant.

\section{REFERENCES}

[1] W. Nelson, “Accelerated Testing: Statistical Models, Test Plans and Analysis,” Wiley, New York, 2004.

[2] O. Aalen, "Nonparametric Inference for a Family of Counting Processes,” Annals of Statistics, Vol. 6, No. 4, 1978, pp. 701-726. doi:10.1214/aos/1176344247

[3] P. K. Andersen, O. Borgan, R. D. Gill and N. Keiding, "Statistical Model Based on Counting Processes," SpringerVerlag, New York, 1993.

[4] T. Fleming and D. Harrington, "Counting Processes and Survival Analysis,” Wiley, New York, 1992.

[5] D. R. Cox and D. Oakes, "The Analysis of Survival Data,” Chapman and Hall, London, 1984.

[6] R. Barlow and E. Scheuer, "Estimation from Accelerated Life Tests,” Technometrics, Vol. 13, No. 1, 1971, pp. 145160.

[7] B. Gnedenko, I. Pavlov and I. Ushakov, "Statistical Reliability Engineering,” John Wiley \& Sons Ltd., New York, 1999.

[8] R. Hogg and A. Craig, "Introduction to Mathematical Statistics,” John Wiley \& Sons Ltd., New York, 1995.

[9] W. Q. Meeker and L. A. Escobar, "Statistical Methods for Reliability Data,” John Wiley \& Sons Ltd., New York, 1998. 